\title{
Combination of yttrium-90 radioembolization with stereotactic body radiation therapy in the treatment of portal vein tumor thrombosis
}

\author{
Jason Liu', Colton Ladbury ${ }^{1}$, Arya Amini', Scott Glaser ${ }^{1}$, Jonathan Kessler ${ }^{2}$, Aram Lee ${ }^{2}$, Yi-Jen Chen ${ }^{1}$ \\ ${ }^{1}$ Department of Radiation Oncology, City of Hope National Medical Center, Duarte, CA, USA \\ ${ }^{2}$ Department of Interventional Radiology, City of Hope National Medical Center, Duarte, CA, USA
}

Received: February 13, 2021

Revised: May 5, 2021

Accepted: May 7, 2021

Correspondence:

Yi-Jen Chen

Department of Radiation Oncology,

City of Hope National Medical Center,

1500 E Duarte Rd, Duarte 91010, USA

Tel: +1-626-218-2972

Fax: +1-626-218-5534

E-mail: yichen@coh.org

ORCID:

https://orcid.org/0000-0001-8022-3316

Purpose: Portal vein tumor thrombosis (PVT) from cancer involving the liver carries a dismal prognosis, with median overall survival (OS) ranging from 2 to 5 months. While treatment with yttrium-90 $\left({ }^{90} \mathrm{Y}\right)$ radioembolization alone may improve outcomes, overall prognosis remains poor. We hypothesize that the combination of ${ }^{90} \mathrm{Y}$ radioembolization to the parenchymal component of the tumor and stereotactic body radiation therapy (SBRT) to the vascular component is a safe and effective means of improving outcomes.

Materials and Methods: A single center retrospective review identified 12 patients with cancers involving the liver who received both ${ }^{90} \mathrm{Y}$ radioembolization and SBRT to the PVTT between May 2015 to August 2020. Primary endpoint was the 90-day toxicity rate by the Common Terminology Criteria for Adverse Events version 5.0. Secondary endpoints were the best response rate based on the Response Evaluation Criteria in Solid Tumors v1.1, local control rate, portal vein (PV) patency rate, and median OS.

Results: Patients received a median ${ }^{90} \mathrm{Y}$ dose of $104.3 \mathrm{~Gy}$ (range, 83.3 to $131.7 \mathrm{~Gy}$ ) and a median 5-fraction SBRT dose of 32.5 Gy (range, 27.5 to $50 \mathrm{~Gy}$ ). There were no late toxicities reported, and only 7 acute grade 1 toxicities reported: elevation of liver function tests (17\%), nausea (17\%), fatigue (17\%), and esophagitis (8\%). Local control was 83\%. 58\% of patients had a patent PV after treatment. With a median follow-up time of 28 months, 1-year OS was 55\% with a median OS of 14 months.

Conclusion: Combination ${ }^{90} \mathrm{Y}$ radioembolization and SBRT appears to be safe and effective in the treatment of PVTT. Larger prospective studies are warranted to better evaluate this combination treatment approach.

Keywords: Liver, Portal vein, Hepatocellular carcinoma, Therapeutic embolization, Stereotactic body radiotherapy

\section{Introduction}

Portal vein tumor thrombosis (PVTT) is a common complication of tumors involving the liver, occurring in about 40\%-60\% of cases of hepatocellular carcinoma (HCC) [1]. PVTT is commonly associated with portal vein (PV) hypertension, impaired liver function, and liver failure. Prognosis of PVTT is poor, with median survival ranging from 2 to 5 months with best supportive care [2,3]. There is currently no widely accepted consensus for the management of PVTT. Treatment options include systemic therapy, surgery, arterially directed therapies such as radioembolization, or external beam radiation. The most evident survival advantages can be attributed to

Copyright (C) 2021 The Korean Society for Radiation Oncology

This is an Open Access article distributed under the terms of the Creative Commons Attribution Non-Commercial License (http://creativecommons.org/licenses/by-nc/4.0/) which permits unrestricted non-commercial use, distribution, and reproduction in any medium, provided the original work is properly cited. 
surgery, with a median overall survival (OS) up to 37 months, although many patients with advanced cancer are poor surgical candidates [4-6].

Yttrium-90 $\left({ }^{90} \mathrm{Y}\right)$ radioembolization is one viable treatment option in patients unable to undergo surgery. However, objective response rates with ${ }^{90} \mathrm{Y}$ radioembolization alone in patients with PVTT are approximately 50\% [7]. There is growing evidence that stereotactic body radiation therapy (SBRT) is also a potentially viable option for PVT, although SBRT is rarely used alone to cover the entire area of disease in the liver [8-11]. Here, we hypothesize that the combination of ${ }^{90} \mathrm{Y}$ radioembolization to the parenchymal component of the tumor and SBRT to the vascular invasion component is a safe and effective means of improving outcomes.

\section{Materials and Methods}

\section{Data collection}

We retrospectively identified 12 patients with cancers involving the liver and associated PVIT who received both ${ }^{90} \mathrm{Y}$ radioembolization and SBRT to the PVTT between May 2015 and August 2020. The Institutional Review Board of City of Hope National Medical Center (No. 21240) approval was obtained for this study. The informed consent was waived as this was a retrospective study. Demographic, clinical, and treatment factors were recorded. Primary endpoints were treatment complication rate and toxicity rate by the Common Terminology Criteria for Adverse Events (CTCAE) version 5.0. All toxicities $\leq 3$ months (90 days) after treatment completion were considered acute, and all others were considered late. Toxicities were reported during weekly on treatment visits and 1-3 month follow-up visits by the attending or resident physician. When quantifying hepatic impairment, the following criteria were used based on the CTCAE v5.0: grade 1, alanine aminotransferase/aspartate aminotransferase (ALT/AST) 1-3.0 times the upper limit of normal (ULN) or bilirubin 1-1.5 times ULN; grade 2, ALT/AST 3-5 times ULN or bilirubin 1.5-3 times ULN; grade 3, ALT/AST 5-20 times ULN or bilirubin 3-10 times ULN; grade 4, ALT/AST > 20 times ULN or bilirubin > 10 times ULN. For the purposes of our analysis, we chose to use the term "elevated liver function tests (LFTs)" to capture this toxicity. Toxicity data is recorded for all patients using a "toxicity assessment" smartsheet on the Epic electronic medical record system. This is a regular part of our workflow and is almost always filled out.

Secondary endpoints were the best response rate, PV patency rate, 1-year OS rate, and median OS. Best response rates were determined based on the Response Evaluation Criteria in Solid Tumors criteria (RECIST v1.1). Treatment response was evaluated based on the vascular component of treatment. A complete response (CR) was defined as no tumor within the PV, a partial response (PR) was defined as decreased tumor within the PV, stable disease (SD) was defined as stable tumor within the PV, and progressive disease (PD) was defined as tumor growing within the PV. PV patency was determined based on radiologist report and viewing the scan for confirmation. 1-year OS rate was based on the time from start of SBRT to the time of death or most recent follow-up.

\section{Treatment}

All patients were reviewed in a weekly multidisciplinary tumor board dedicated to upper gastrointestinal malignancies. It is our general principle to use ${ }^{90} \mathrm{Y}$ radioembolization followed by SBRT to treat patients all patients who are not surgical candidates with HCC with PVTT. Reasons that may preclude a patient from surgery include co-morbidities such as cirrhosis and diffuse or multifocal disease. Our approach has been to deliver ${ }^{90} \mathrm{Y}$ radioembolization to treat the parenchymal component of the tumor followed by SBRT to the vascular invasion component of the tumor. SBRT was typically planned from the start. When we first started this combination treatment modality, we waited until after 90 days after ${ }^{90} \mathrm{Y}$ radioembolization to initiate SBRT because we typically do not see the maximum effect of ${ }^{90} \mathrm{Y}$ until after 90 days. However, our experience has progressively shown that PVTT does not respond to ${ }^{90} \mathrm{Y}$ alone, so we have been more routinely offering SBRT sooner within 1-3 months and not wait 90 days to evaluate response. Fig. 1 illustrates the overall timeline for ${ }^{90} Y$ radioembolization and SBRT treatment planning and evaluation.

For ${ }^{90} \mathrm{Y}$ radioembolization, all patients underwent a standardized pretreatment workup consisting of clinical evaluation, laboratory and imaging assessment, a mapping angiographic procedure, and technetium-99 macroaggregated albumin scintigraphy. ${ }^{90} \mathrm{Y}$-microspheres (Sirtex, Woburn, MA, USA) were administered to the appropriate segmental or lobar feeding arteries. Our institutional practice is to give the ${ }^{90} \mathrm{Y}$ activity that will result in the intended volume of the liver receiving between 80 to $150 \mathrm{~Gy}$. There were no whole liver ${ }^{90} Y$ treatments in this cohort. After treatment, patients routinely underwent a limited positron emission tomography/computed tomography (PET/CT) scan to visualize areas of inflammation to approximate ${ }^{90} \mathrm{Y}$ uptake.

For SBRT treatments, all patients underwent a four-dimensional $\mathrm{CT}$ simulation scan. Patients received IV contrast and were immobilized using a VacLoc. In patients with adequate lung function, end expiratory breath holding was used to minimize breathing-related liver motion, otherwise respiratory gating was used. The gross tumor volume (GTV) defined as the primary lesion in the PV was delineated using the CT simulation image and/or fused magnetic resonance imaging (MRI) scan. For patients undergoing respiratory 


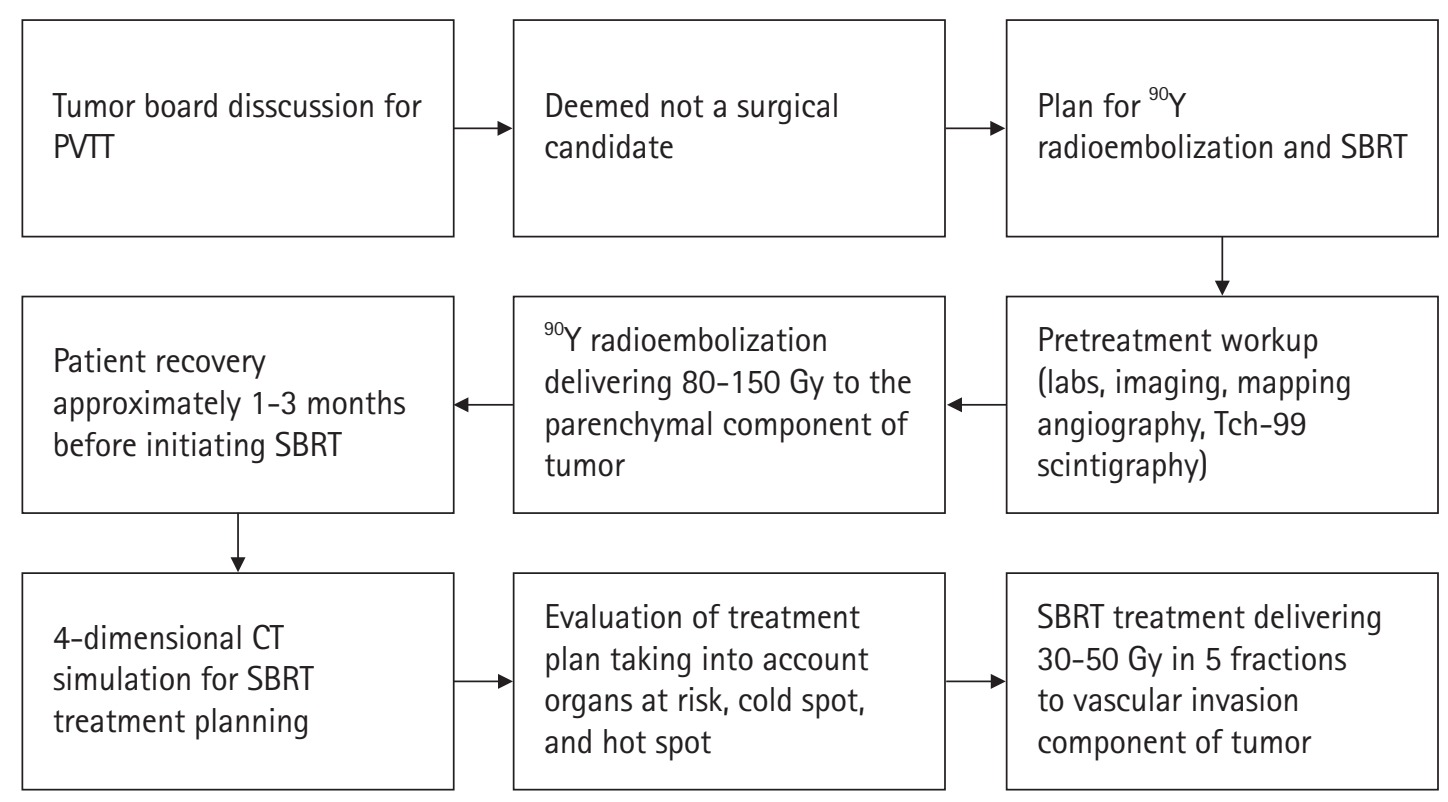

Fig. 1. Timeline of yttrium-90 $\left({ }^{90} \mathrm{Y}\right)$ radioembolization and stereotactic body radiation therapy (SBRT) planning and evaluation. PVTT, portal vein tumor thrombosis; CT, computed tomography.

gating, the internal target volume (ITV) was delineated similarly, making sure to include the primary lesion in all phases of breathing during which the patient will be treated. The planning target volume (PTV) was defined as the GTV or ITV plus a 5-mm radial margin. Dose was prescribed to $95 \%$ of the PTV. For treatment planning for 5-fraction SBRT, dose constraints commonly used were liver $\mathrm{V}_{15}<700 \mathrm{~mL}$, duodenum $\mathrm{D}_{\text {max }}<32 \mathrm{~Gy}$, esophagus $\mathrm{D}_{\max }<35$ Gy, and spinal cord $D_{\max }<30 \mathrm{~Gy}$. To limit hot spots, max dose within the target was limited to $<110 \%$. Unfortunately, there was no method to account for the prior radiation dose delivered to the normal liver via ${ }^{90} Y$ radioembolization. Generally, SBRT to the vascular invasion component of the tumor contributed very little radiation to the normal liver given its focused target area. However, if there is concern for normal liver receiving too much radiation from both treatments, the limited PET/CT scan obtained after ${ }^{90} \mathrm{Y}$ can be used to crop the SBRT treatment volume off of overlapping liver volumes. For treatment delivery, image guidance with cone beam CT was used to verify positioning before each treatment. Fiducial markers were used in select patients. SBRT was delivered every other day up to a total of 5 fractions.

\section{Statistical analysis}

Descriptive statistics such as medians for continuous variables and frequencies for categorical variables were used to describe patient characteristics. Median follow-up time (from the completion of SBRT) was calculated with the reverse survival method. Survival analyses were performed using Kaplan-Meier analysis and Cox re- gression for univariate analysis. All analyses were done using the statistical software SPSS version 26 (IBM, Armonk, NY, USA). All tests used a significance level $<0.05$.

\section{Results}

\section{Patient demographics}

A total of 12 patients received the combination of ${ }^{90} \mathrm{Y}$ and SBRT for PVTT from May 2015 to August 2020. Baseline patient characteristics were gathered and shown in Table 1. None of the patients had any prior treatment such as surgical resection, liver transplant, or systemic therapy. Most patients were 65 years or older $(68 \%)$, male (68\%), had a primary diagnosis of HCC (75\%), the Eastern Cooperative Oncology Group (ECOG) performance status of 0 (58\%), Child-Pugh class A (75\%), with PVIT in the left or right PV (75\%).

Patients received a median ${ }^{90} \mathrm{Y}$ dose of $104.3 \mathrm{~Gy}$ (range, 83.3 to $131.7 \mathrm{~Gy}$ ) to a median volume of $846 \mathrm{~mL}$ (range, 675 to $1,068 \mathrm{~mL}$ ). Most patients had a 1- to 3-month interval between ${ }^{90} \mathrm{Y}$ and SBRT (75\%). Patients received a median SBRT dose of 32.5 Gy in 5 fractions (range, 27.5 to $50 \mathrm{~Gy}$ in 5 fractions) to a median volume of $91.3 \mathrm{~mL}$ (range, 14.7 to $623.2 \mathrm{~mL}$ ). The most common fractionation was $30 \mathrm{~Gy}$ in 5 fractions. There were three patients with progression of parenchymal disease in the time between ${ }^{90} \mathrm{Y}$ and SBRT. These three patients with disease progression had a larger PTV volume (range, 266.1 to $623.2 \mathrm{~mL}$ ) than patients who did not have disease progression (range, 14.7 to $192.4 \mathrm{~mL}$ ) to cover additional areas of parenchymal disease. 
Table 1. Summary of patient demographics, clinical factors, and treatment variables $(n=12)$

\begin{tabular}{|c|c|}
\hline & Value \\
\hline Age (yr) & $65.5(56-83)$ \\
\hline$<65$ & $5(42)$ \\
\hline$\geq 65$ & $7(58)$ \\
\hline \multicolumn{2}{|l|}{ Sex } \\
\hline Male & $7(58)$ \\
\hline Female & $5(42)$ \\
\hline \multicolumn{2}{|l|}{ Race } \\
\hline White & $5(42)$ \\
\hline Hispanic & $2(17)$ \\
\hline Asian & $5(42)$ \\
\hline \multicolumn{2}{|l|}{ Diagnosis } \\
\hline $\mathrm{HCC}$ & $9(75)$ \\
\hline Cholangiocarcinoma & $2(17)$ \\
\hline Neuroendocrine liver metastasis & $1(8)$ \\
\hline \multicolumn{2}{|l|}{ ECOG performance status } \\
\hline 0 & $7(58)$ \\
\hline 1 & $4(33)$ \\
\hline 2 & $1(8)$ \\
\hline \multicolumn{2}{|l|}{ Child-Pugh classification } \\
\hline A & $9(75)$ \\
\hline B & $3(25)$ \\
\hline C & $0(0)$ \\
\hline \multicolumn{2}{|l|}{ Location of PVTT } \\
\hline Segmental branch & $2(17)$ \\
\hline Left/right PV & $9(75)$ \\
\hline Main PV & $1(8)$ \\
\hline${ }^{90} \mathrm{Y}$ dose (Gy) & $104.3(83.3-131.7)$ \\
\hline${ }^{90} \mathrm{Y}$ volume $(\mathrm{mL})$ & $846(675-1,068)$ \\
\hline Five-fraction SBRT dose (Gy) & $32.5(27.5-50)$ \\
\hline$<30$ & $3(25)$ \\
\hline $30-35$ & $4(33)$ \\
\hline $40-45$ & $3(25)$ \\
\hline$\geq 50$ & $2(17)$ \\
\hline \multicolumn{2}{|l|}{ Days between ${ }^{90} \mathrm{Y}$ and SBRT } \\
\hline$<30$ & $1(8)$ \\
\hline $30-60$ & $5(42)$ \\
\hline $60-90$ & $2(17)$ \\
\hline$>90$ & $4(33)$ \\
\hline PTV volume (mL) & $91.3(14.7-623.2)$ \\
\hline$<50$ & $2(17)$ \\
\hline $50-100$ & $5(42)$ \\
\hline $100-250$ & $2(17)$ \\
\hline $250-500$ & $2(17)$ \\
\hline$>500$ & $1(8)$ \\
\hline \multicolumn{2}{|l|}{ Previous treatments } \\
\hline Surgical resection & $0(0)$ \\
\hline Liver transplant & $0(0)$ \\
\hline Systemic therapy & $0(0)$ \\
\hline \multicolumn{2}{|l|}{ Maintenance therapy afterwards } \\
\hline Yes & $7(58)$ \\
\hline No & $5(42)$ \\
\hline
\end{tabular}

Values are presented as median (range) or number (\%).

HCC, hepatocellular carcinoma; ECOG, Eastern Cooperative Oncology Group; PVT, portal vein tumor thrombosis; PV, portal vein; ${ }^{90} \mathrm{Y}$, yttrium-90; SBRT, stereotactic body radiation therapy; PTV, planning target volume.
Most patients were treated using end expiration breath-hold (75\%), and the remaining were treated using respiratory gating. Fifty-eight percent of patients received some form of maintenance therapy after local treatment such as sorafenib, lenvatinib, bevacizumab, or nivolumab.

\section{SBRT related toxicities}

A summary of SBRT related toxicities can be seen in Table 2. Of the 12 patients in this study, 17\% developed acute grade 1 elevation of LFTs, 17\% developed acute grade 1 nausea, 17\% developed acute grade 1 fatigue, and 8\% developed acute grade 1 esophagitis. The total rate of grade 1 toxicity was 59\%. There were no grade $2+$ acute toxicities or late toxicities or treatment complications reported. As mentioned above, the interval between ${ }^{90} \mathrm{Y}$ and SBRT was shortened from over 3 months to 1-3 months after our experience showed PVTT rarely responds to ${ }^{90} \mathrm{Y}$ alone. After shortening the interval, we observed no increase in acute toxicities in patients treated with a 1- to 3-month interval (5/8 patients) vs. patients treated with >3-month interval (2/4 patients), although sample sizes are too small to draw any conclusions. Individual patient level demographics and toxicities can be seen in Table 3.

\section{Response rates and survival}

Based on RECIST criteria v1.1, the CR rate was $8 \%$, the PR rate was $42 \%$, the SD rate was 33\%, and the PD rate was $17 \%$. After shortening the interval between ${ }^{90} \mathrm{Y}$ and SBRT from over 3 months to 1-3 months, we observed no difference in response rates in patients treated with a 1- to 3-month interval (7/8 patients with $\mathrm{CR}+\mathrm{PR}+\mathrm{SD})$ vs. patients treated with $>3$-month interval $(3 / 4$ pa-

Table 2. Summary of toxicities, response rates, and PV patency

\begin{tabular}{ll}
\hline & Value \\
\hline SBRT related toxicities & \\
Acute grade 1 elevated LFTs & $2(17)$ \\
Acute grade 1 nausea & $2(17)$ \\
Acute grade 1 fatigue & $2(17)$ \\
Acute grade 1 esophagitis & $1(8)$ \\
Late toxicities & $0(0)$ \\
Best response by RECIST v1.1 & \\
Complete response & $1(8)$ \\
Partial response & $5(42)$ \\
Stable disease & $4(33)$ \\
Progressive disease & $2(17)$ \\
PV patency after treatment & \\
Yes & $7(58)$ \\
No & $5(42)$
\end{tabular}

Values are presented as number of patients (\%).

PV, portal vein; SBRT, stereotactic body radiation therapy; LFT, liver function test; RECIST, Response Evaluation Criteria in Solid Tumors. 
tients with $\mathrm{CR}+\mathrm{PR}+\mathrm{SD}$ ), although sample sizes are too small to draw any conclusions. The local control rate (CR+PR+SD) was 83\% (Fig. 2). On follow-up imaging, 58\% patients had a patent PV. With a median follow-up of 29 months, 1-year OS was 55\% and median OS was 14 months (Fig. 3). A summary of response rate, PV patency rate, and 1-year OS rate can be seen in Table 2. Individual patient level demographics, response rates, and 1-year OS rates can be seen in Table 3.

A univariate analysis was performed to determine predictors for improved OS (Table 4). The only variable to be significantly associated with improved OS was treatment response, dichotomized into $C R+P R+S D$ versus PD $(p<0.001)$. Age, sex, diagnosis, performance status, Child-Pugh, location of PVTT, ${ }^{90} \mathrm{Y}$ dose, total SBRT dose, PTV volume, interval between ${ }^{90} \mathrm{Y}$ and SBRT, and receiving maintenance therapy after local treatment were not significantly associated with OS. Fig. 3 shows the Kaplan-Meier curve comparing OS for responders to ${ }^{90} \mathrm{Y}$-SBRT versus non-responders. Median OS for treatment responders was 33 months versus 2 months for treatment non-responders $(p<0.001)$.

\section{Discussion and Conclusion}

There is currently insufficient data supporting the safety of combination therapy. Previous consensus statements have stated previous ${ }^{90} \mathrm{Y}$ radioembolization as a relative contraindication to SBRT due to concern of the liver receiving too much radiation [12]. However, there has been a growing number of radiation oncologists comfortable with offering SBRT after ${ }^{90} Y$ radioembolization based on anecdotal evidence that it can be delivered safely. In this retrospective analysis, we show that the combination of ${ }^{90} \mathrm{Y}$ and SBRT is safe and well tolerated in the treatment of PVIT.

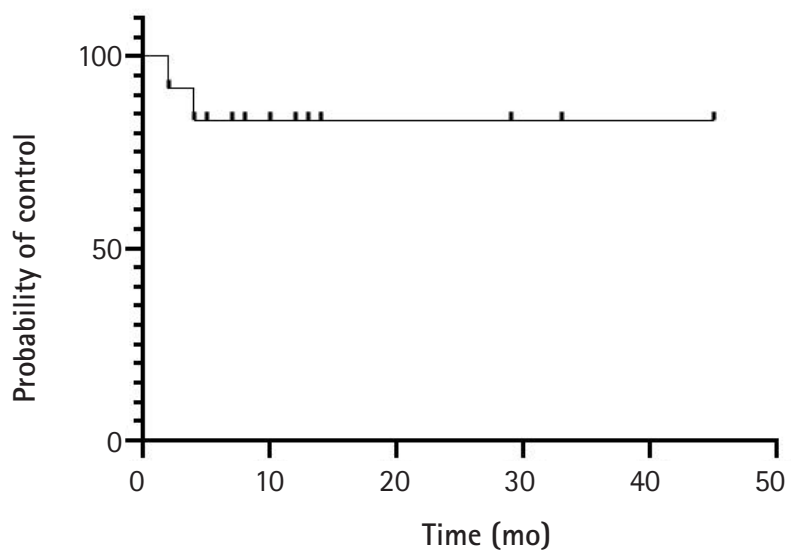

Fig. 2. Local control for all patients.

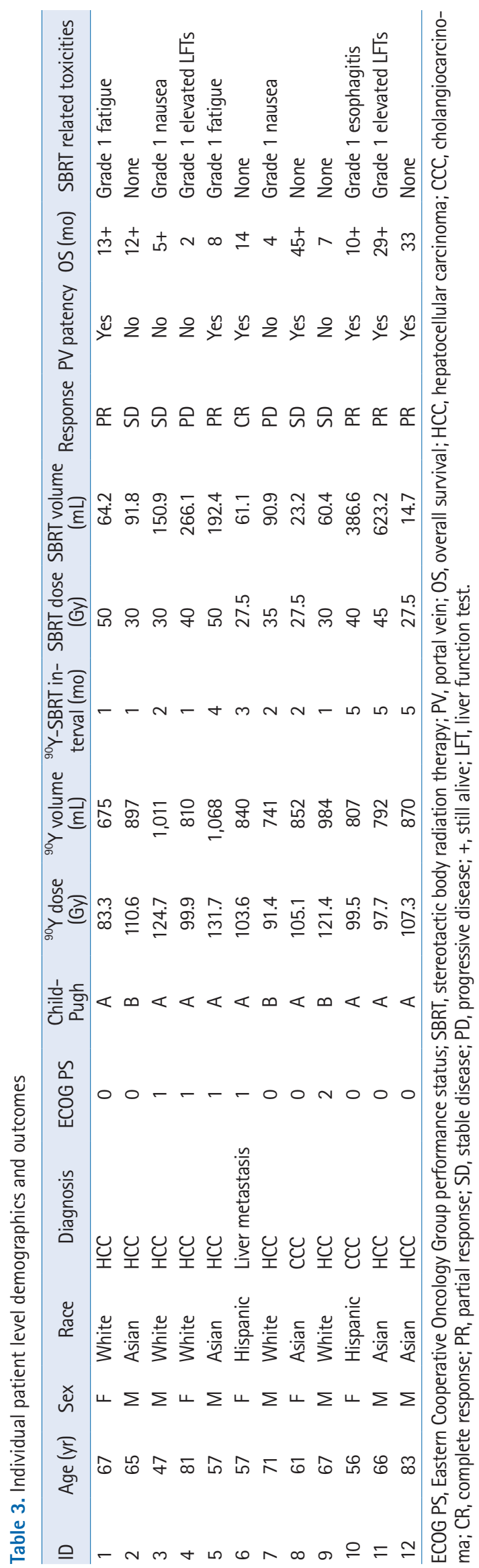



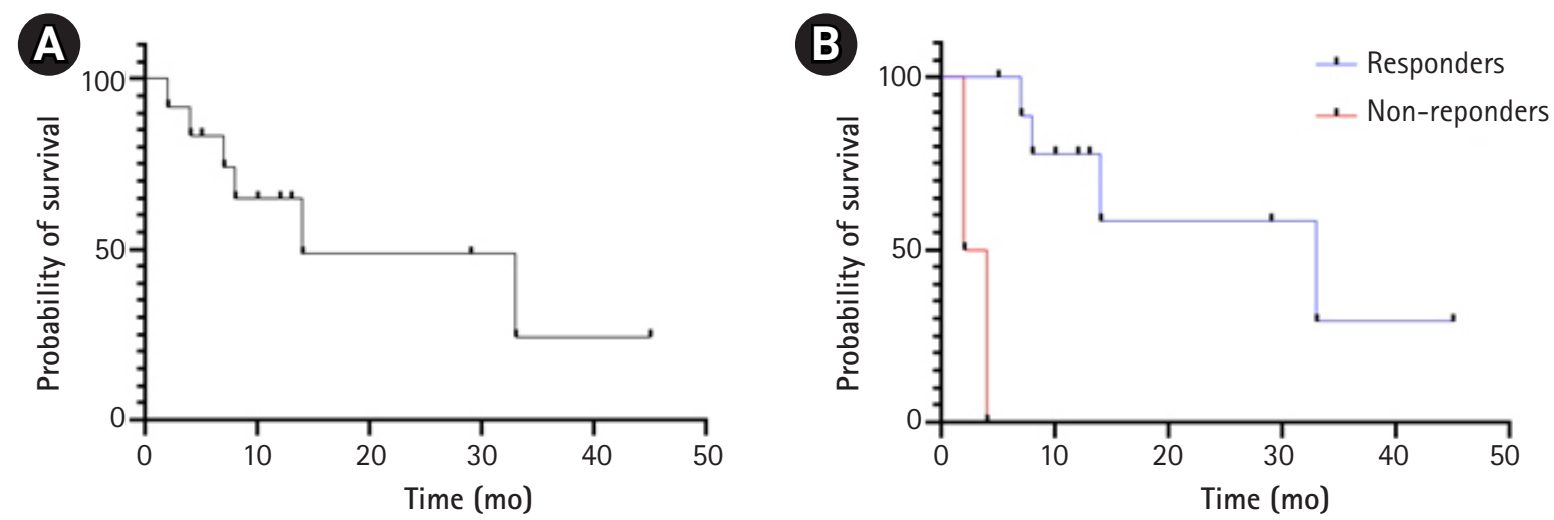

Fig. 3. (A) Kaplan-Meier curve for all patients. Median overall survival (OS) was 14 months. (B) Kaplan-Meier curve comparing ${ }^{90} Y-S B R T$ responders (CR+PR+SD) versus non-responders (PD). Median OS for responders was 33 months versus 2 months for non-responders ( $p<0.001)$. SBRT, stereotactic body radiation therapy; ${ }^{90} \mathrm{Y}$, yttrium-90; CR, complete response; PR, partial response; $S D$, stable disease; PD, progressive disease.

Table 4. Univariate analysis for predictors of overall survival

\begin{tabular}{lc}
\hline & p-value \\
\hline Diagnosis & \\
$\quad$ Cholangiocarcinoma vs. HCC & 0.832 \\
$\quad$ Neuroendocrine liver metastasis vs. HCC & 0.863 \\
Age (yr) & \\
$\quad \geq 65$ vs. <65 & 0.511 \\
Sex & 0.888 \\
$\quad$ Male vs. female & \\
Child-Pugh classification & 0.358 \\
$\quad$ B vs. A & \\
Dose fractionation & \\
$30-35$ Gy vs. <30 Gy in 5 fx & 0.915 \\
$\quad 40-45$ Gy vs. <30 Gy in $5 \mathrm{fx}$ & 0.819 \\
$50+$ Gy vs. <30 Gy in $5 \mathrm{fx}$ & 0.781 \\
PTV volume (mL) & \\
$50-100$ vs. <50 & 0.866 \\
100-250 vs. <50 & 0.736 \\
$250-500$ vs. <50 & 0.607 \\
$500+$ vs. <50 & 0.429 \\
Treatment response & \\
Responders (SD+PR+CR) vs. non-responders (PD) & 0.001 \\
\hline
\end{tabular}

$\mathrm{HCC}$, hepatocellular carcinoma; PTV, planning target volume; $\mathrm{CR}$, complete response; $\mathrm{PR}$, partial response; $\mathrm{SD}$, stable disease; $\mathrm{PD}$, progressive disease.

Recently, published data has suggested that the combination of arterial therapy and SBRT can be performed safely with good shortterm outcomes. A randomized clinical trial by Yoon et al. [13], prospectively compared transarterial chemoembolization (TACE) plus external beam radiotherapy versus sorafenib in HCC with macroscopic vascular invasion. It was found that first-line treatment with TACE plus SBRT was well tolerated and had improved progression-free survival (PFS), objective response rate, and OS compared to sorafenib. Hardy-Abeloos et al. [14] retrospectively identified 68 patients with HCC who received SBRT after TACE and 31 patients who received SBRT after ${ }^{90} \mathrm{Y}$ radioembolization. The rate of grade $\geq 3$ toxicities were similar between TACE-SBRT and ${ }^{90} \mathrm{Y}$-SBRT (13\% vs. 9.4\%). One-year LC rate for ${ }^{90} Y-S B R T$ was $97 \%$, and the median OS for ${ }^{90} \mathrm{Y}$-SBRT was not reached after 18 months. In the ${ }^{90} \mathrm{Y}$-SBRT arm, 14 patients (45\%) achieved a CR, while 5 patients (16\%) achieved a PR. Fourteen patients (14\%) had PVTT (8 in the TACESBRT arm and 6 in the ${ }^{90} \mathrm{Y}-\mathrm{SBRT}$ arm), but this study did not specifically analyze this population.

The current study supports this previous report that combination ${ }^{90} Y$ radioembolization and SBRT can be performed safely. In the current study, no acute or late grade $\geq 3$ toxicities were identified. Several factors may account for this. Firstly, the current study had a lower median SBRT dose of 32.5 Gy in 5 fractions compared to a median SBRT dose of 40 Gy in 5 fractions in the previous study. Furthermore, our median PTV volume was smaller at $91.3 \mathrm{~mL}$ compared to $103.1 \mathrm{~mL}$. Also, less parenchyma was included in the PTV since the SBRT was only targeting the PVTT. Our sample size was also much smaller, which may account for the lack of patients reporting grade $\geq 3$ toxicities.

The efficacy of combination therapy in the current study is also encouraging. One patient (8\%) who achieved a CR and 5 patients $(42 \%)$ achieved a PR in the current study. This is lower than seen in the previous report of combination therapy, where CR and PR were 45\% and 16\% respectively. Similarly, the current study's 1-year OS rate was also lower at 55\% with a median OS of 14 months compared to $70 \%$ with a median OS not reached after 18 months in the previous trial. However, these findings are expected given the overall worse response rates and survival in patients with PVTT (Fig. 4).

Several previous studies have analyzed the outcomes of combination therapy or SBRT alone in patients with PVTT and demonhttps://doi.org/10.3857/roj.2021.00213 


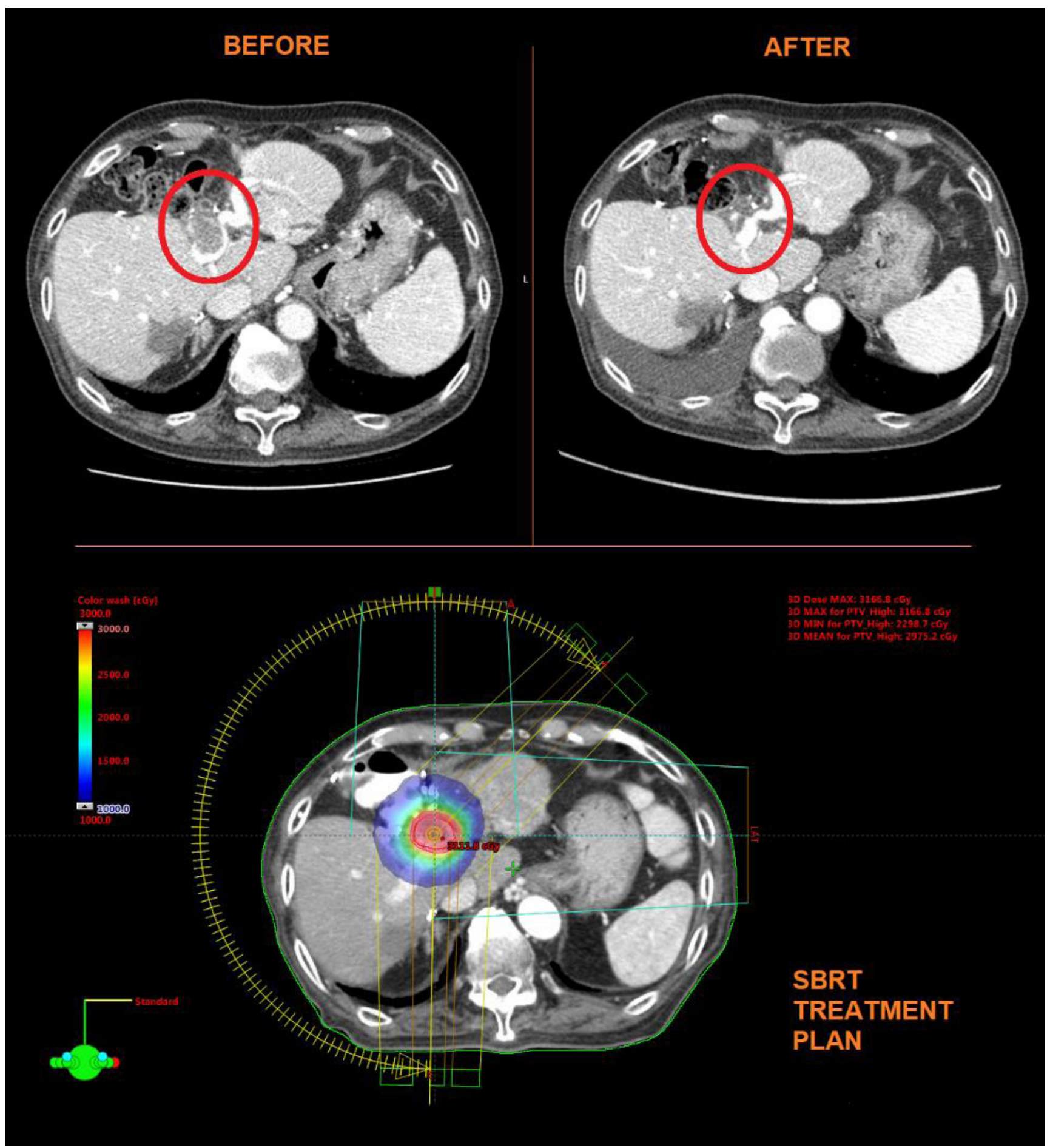

Fig. 4. Example of a SBRT plan to the PVTT and near CR after treatment. Patient \#12 was an 83-year-old Asian male with hepatocellular carcinoma who received surgery, transarterial chemoembolization, and sorafenib, but progressed with worsening PVTT. Patient was treated with ${ }^{90} Y$ radioembolization 107.3 Gy followed by SBRT 27.5 Gy in 5 fractions. Patient had near CR 3 months after SBRT. SBRT, stereotactic body radiation therapy; PVT, portal vein tumor thrombosis; CR, complete response.

strate similar efficacy to the current report. A retrospective study of 37 patients with HCC with PVTT received TACE followed by SBRT found a CR rate of $16.2 \%$ and a PR rate of 54\% [15]. These values are slightly higher than those of our study (8\% and $42 \%$, respec- tively). Furthermore, the 1-year OS rate was $54.1 \%$ with a median OS of 15 months, which is closely in line with our study (55\% with median OS of 14 months).

Another retrospective study of 24 patients with HCC with PVTT 
treated with SBRT found a CR rate of 8.3\% and a PR rate of $45.8 \%$ [16]. Another retrospective study of 70 patients with HCC with PVTT treated with SBRT found a 1-year OS rate of $40 \%$ with a median OS of 10 months [17]. The current study demonstrated a 1 -year OS rate of $55 \%$ with a median OS of 14 months.

In the univariate analysis, only response to ${ }^{90} \mathrm{Y}-\mathrm{SBRT}$ was associated with improved $O S$, with patients who achieved a $C R$, PR, or SD after treatment had a significantly longer median OS compared to those who had PD (33 months vs. 2 months; $p<0.001$ ) (Fig. 3). This is consistent with two previous studies. One retrospective study examining SBRT for PVTT found that patients who achieved a $C R$, PR, or SD had a median OS of 13 months versus 4 months for patients who had PD [17]. Another retrospective study examining SBRT for PVTT found that patients with controlled disease had a median OS of 18.8 months versus 7.8 months in those with local progression [18]. This is reasonable, as PD can result in complete occlusion of the PV, eventually leading to liver failure. These two studies also identified other predictors for OS not found in our study, including PVTT location, a biologically effective dose (BED) $>100 \mathrm{~Gy}$, and a smaller GTV volume $[17,18]$. The absence of these associations in the current study may be due to the small sample size of the current study.

While the results of our study are promising, there are several limitations. The analysis is retrospective and single institution, so results will need to be prospectively validated. Furthermore, the sample size of 12 makes it difficult to perform any meaningful statistical analysis examining predictors of improved response or survival. However, as a feasibility study, our goal is to simply report that ${ }^{90} Y$-SBRT for PVTT is safe and can offer adequate LC and improved OS. Larger prospective studies are warranted to better evaluate this combined treatment modality and determine what factors may predict for improved outcomes.

Despite these limitations, our study shows that the combination of ${ }^{90} \mathrm{Y}$ radioembolization and SBRT is a safe and well-tolerated treatment for PVIT. There were no recorded grade 2+ toxicities. Successful treatment may result in improved survival. As always, having a multi-disciplinary approach to treating advanced liver cancer with PVTT is the best approach to maximizing patients' quality of life and survival.

\section{Conflict of Interest}

No potential conflict of interest relevant to this article was reported.

\section{References}

1. Takizawa D, Kakizaki S, Sohara N, et al. Hepatocellular carcinoma with portal vein tumor thrombosis: clinical characteristics, prognosis, and patient survival analysis. Dig Dis Sci 2007;52:3290-5.

2. Siegel RL, Miller KD, Jemal A. Cancer statistics, 2020. CA Cancer J Clin 2020;70:7-30.

3. Chen W, Zheng R, Baade PD, et al. Cancer statistics in China, 2015. CA Cancer J Clin 2016;66:115-32.

4. Torzilli G, Belghiti J, Kokudo N, et al. A snapshot of the effective indications and results of surgery for hepatocellular carcinoma in tertiary referral centers: is it adherent to the EASL/AASLD recommendations? An observational study of the HCC East-West study group. Ann Surg 2013;257:929-37.

5. Cerrito $L$, Annicchiarico $B E$, lezzi $R$, Gasbarrini $A$, Pompili $M$, Ponziani FR. Treatment of hepatocellular carcinoma in patients with portal vein tumor thrombosis: beyond the known frontiers. World J Gastroenterol 2019;25:4360-82.

6. Shi J, Lai EC, Li N, et al. Surgical treatment of hepatocellular carcinoma with portal vein tumor thrombus. Ann Surg Oncol 2010;17:2073-80.

7. Somma F, Stoia V, Serra N, D'Angelo R, Gatta G, Fiore F. Yttrium-90 trans-arterial radioembolization in advanced-stage HCC: the impact of portal vein thrombosis on survival. PLoS One 2019;14:e216935.

8. Xi M, Zhang L, Zhao L, et al. Effectiveness of stereotactic body radiotherapy for hepatocellular carcinoma with portal vein and/ or inferior vena cava tumor thrombosis. PLoS One 2013;8:e638 64.

9. Hannan R, Margulis V, Chun SG, et al. Stereotactic radiation therapy of renal cancer inferior vena cava tumor thrombus. Cancer Biol Ther 2015;16:657-61.

10. Choi BO, Choi IB, Jang HS, et al. Stereotactic body radiation therapy with or without transarterial chemoembolization for patients with primary hepatocellular carcinoma: preliminary analysis. BMC Cancer 2008:8:351.

11. Lin CS, Jen YM, Chiu SY, et al. Treatment of portal vein tumor thrombosis of hepatoma patients with either stereotactic radiotherapy or three-dimensional conformal radiotherapy. Jpn J Clin Oncol 2006;36:212-7.

12. Hong K, Akinwande O, Bodei $L$, et al. ACR-ABS-ACNM-ASTROSIR-SNMMI practice parameter for selective internal radiation therapy or radioembolization for treatment of liver malignancies. Brachytherapy 2021;20:497-511.

13. Yoon SM, Ryoo BY, Lee SJ, et al. Efficacy and safety of transarterial chemoembolization plus external beam radiotherapy vs 
sorafenib in hepatocellular carcinoma with macroscopic vascular invasion: a randomized clinical trial. JAMA Oncol 2018;4:661-9.

14. Hardy-Abeloos C, Lazarev S, Ru M, et al. Safety and efficacy of liver stereotactic body radiation therapy for hepatocellular carcinoma after segmental transarterial radioembolization. Int J Radiat Oncol Biol Phys 2019;105:968-76.

15. Kang J, Nie Q, DU R, et al. Stereotactic body radiotherapy combined with transarterial chemoembolization for hepatocellular carcinoma with portal vein tumor thrombosis. Mol Clin Oncol 2014;2:43-50.
16. Jeong BK, Choi HS, Kang KM, et al. Stereotactic-body radiotherapy for portal vein tumor thrombosis in hepatocellular carcinoma patients. J Clin Oncol 2018;36(4_suppl):442.

17. Shui $Y$, Yu W, Ren $X$, et al. Stereotactic body radiotherapy based treatment for hepatocellular carcinoma with extensive portal vein tumor thrombosis. Radiat Oncol 2018;13:188.

18. Scorsetti M, Comito T, Cozzi L, et al. The challenge of inoperable hepatocellular carcinoma (HCC): results of a single-institutional experience on stereotactic body radiation therapy (SBRT). J Cancer Res Clin Oncol 2015;141:1301-9. 